\title{
ОСОБЕННОСТИ ПРОЦЕССОВ ПЫЛЕ-
}

\section{И ДЫМООБРАЗОВАНИЯ ПРИ ГАЗОКИСЛОРОДНОЙ ПРОДУВКЕ КОНВЕРТЕРНОЙ ВАННЫ}

\author{
В. В. Солоненко, Е. В. Протопопов, М. В. Темлянцев, \\ Н. Ф. Якушевич, С. О. Сафонов
}

Сибирский государственный индустриальный университет (Россия, 654007, Кемеровская обл. - Кузбасс, Новокузнецк, ул. Кирова, 42)

Аннотация. Проведено исследование особенностей процессов пыле- и дымообразования при газокислородной продувке конвертерной ванны. Определены основные причины, вызывающие угар металла. Изучены особенности влияния основных параметров процесса на потери металла при пылевыносе и испарении железа в реакционной зоне. Выполнена оценка процесса распыления металла за счет всплывающих пузырей $\mathrm{CO}$, который определяется скоростью их подъема на поверхность ванны. Определены особенности температурного режима реакционной зоны и баланса тепла при добавке топлива к кислородному потоку. Добавка топлива к кислороду позволяет увеличить приход тепла в ванну, при этом уменьшает скорость обезуглероживания. Это способствует уменьшению количества выносимый пыли, образующейся при разрыве и дроблении пленок металла газовыми пузырями. Рассмотрено влияние использования кислорода продуктов горения на окисление примесей металла. На примере продувки углеродистой и легированной стали для прокатных валков показано, что степени разложения $\mathrm{CO}_{2}$ и $\mathrm{H}_{2} \mathrm{O}$ в ванне являются основными характеристиками газокислородной продувки. Эти показатели определяют окислительные и нагревательные свойства дутья. Выполнена оценка изменения суммарного, усвоенного тепла и его потерь с отходящими газами в зависимости от степени разбавления кислородного потока природным газом (метаном). В данных условиях использование погружных факелов горения при изменении окислительной способности факелов позволяет решать различные технологические задачи, в том числе является эффективным способом уменьшения пылевыделения в конвертерном процессе.

Ключевые слова: газокислородная продувка, металл, топливо, реакционная зона, погружные факелы, всплывающие пузыри, пылевынос, дымообразование

Для цитирования: Солоненко В.В., Протопопов Е.В., Темлянцев М.В., Якушевич Н.Ф., Сафонов С.О. Особенности процессов пыле- и дымообразования при газокислородной продувке конвертерной ванны // Известия вузов. Черная металлургия. 2021. Т. 64. № 2. С. 112-121. https://doi.org/10.17073/0368-0797-2021-2-112-121

\section{NATURE OF DUST AND SMOKE GENERATION DURING GAS-OXYGEN BLASTING IN CONVERTER BATH}

\author{
V. V. Solonenko, E. V. Protopopov, M. V. Temlyantsev, \\ N. F. Yakushevich, S. O. Safonov
}

I Siberian State Industrial University (42 Kirova str., Novokuznetsk, Kemerovo Region - Kuzbass 654007, Russian Federation)

Abstract. The article presents the study of the nature of dust and smoke generation during gas-oxygen blasting of a converter bath. The main reasons causing metal waste have been determined. Influence of the process main parameters on metal loss has been studied during dust removal and evaporation of iron in the reaction zone. The authors have estimated the process of metal pulverization due to CO bubbles floating, determined by the rate of their rise to the bath surface. Specifics of temperature regime of the reaction zone and heat balance have been determined when adding fuel to the oxygen flow. Adding fuel to oxygen makes it possible to increase heat input into the bath, while reducing the rate of decarburization. This enables reduction of dust discharge during rupture and crush of metal films by gas bubbles. The effect of combustion products oxygen use on metal impurities oxidation is considered. By the example of blasting carbon and alloyed steel for mill rolls, it has been shown that the degrees of $\mathrm{CO}_{2}$ and $\mathrm{H}_{2} \mathrm{O}$ decomposition in the bath are the main qualities of gas-oxygen blasting. These indicators determine the oxidizing and heating properties of the blast. Assessment of change in total, consumed heat and its losses with exhaust gases, depending on degree of the oxygen flow dilution with natural gas (methane), has been carried out. Under these conditions, use of submersible combustion torches with change in their oxidizing ability makes it possible to solve various technological tasks, including provision of an effective way to reduce dust emission in converter process.

Keywords: gas-oxygen blast, metal, fuel, reaction zone, submerged torches, floating bubbles, dust duscharge, smoke generation

For citation: Solonenko V.V., Protopopov E.V., Temlyantsev M.V., Yakushevich N.F., Safonov S.O. Nature of dust and smoke generation during gasoxygen blasting in converter bath. Izvestiya. Ferrous Metallurgy. 2021, vol. 64, no. 2, pp. 112-121. (In Russ.). https://doi.org/10.17073/0368-07972021-2-112-121 


\section{ВВЕДЕНИЕ}

Одним из перспективных направлений повышения эффективности кислородно-конвертерных процессов является продувка жидкой ванны факелами горения с использованием жидкого или газообразного топлива (рис. 1). Верхняя, верхне-боковая или встречная верхне-донная продувка конвертерной ванны позволяют обеспечить совмещение процессов рафинирования и подогрева металлошихты за счет тепла горения топлива и возможности воздействия факелами на окислительно-восстановительные процессы в ванне, влиять на показатели шлакообразования, а также процессы дымообразования и пылевыделения [1 - 7].

При рассмотрении возможных потерь металла $[2,8]$ при продувке конвертерной ванны следует различать процессы пылевыделения, связанные с выносом из конвертера отходящими газами капель металла, шлака и частиц сыпучих материалов, и дымовыделения при испарении веществ в высокотемпературных реакционных зонах с последующей частичной конденсацией паров в более «холодных» верхних горизонтах рабочего пространства. Интенсивное выделение бурого дыма по ходу продувки является существенной осо- бенностью и недостатком процесса и в значительной степени зависит от температуры в реакционных зонах и интенсивности перемешивания ванны. Содержание пыли, в зависимости от скорости потока отходящих газов (скорости обезуглероживания) и степени вспенивания шлако-металлической эмульсии, может составлять $80-120$ и даже $250-350$ г $\mathrm{M}^{3}$, а потери железа с пылью могут достигать $0,4-1,2 \%$ от массы металлозавалки. При этом потери металла с дымом при продувке кислородом обычно составляют $0,8-1,5 \%$ [2].

В целом определенные на основании практических данных показатели свидетельствуют о довольно значительных потерях металла и снижении выхода жидкой стали при продувке ванны погружными струями, поэтому требуется их дополнительный учет и анализ.

\section{ОСОБЕННОСТИ ПРОЦЕССОВ ПЫЛЕ-}

\section{И ДЫМООБРАЗОВАНИЯ}

К основным причинам, вызывающим повышенный угар металла, можно отнести:

- дробление металла на капли за счет динамической энергии газовых струй с последующей эжекцией капель в струю;

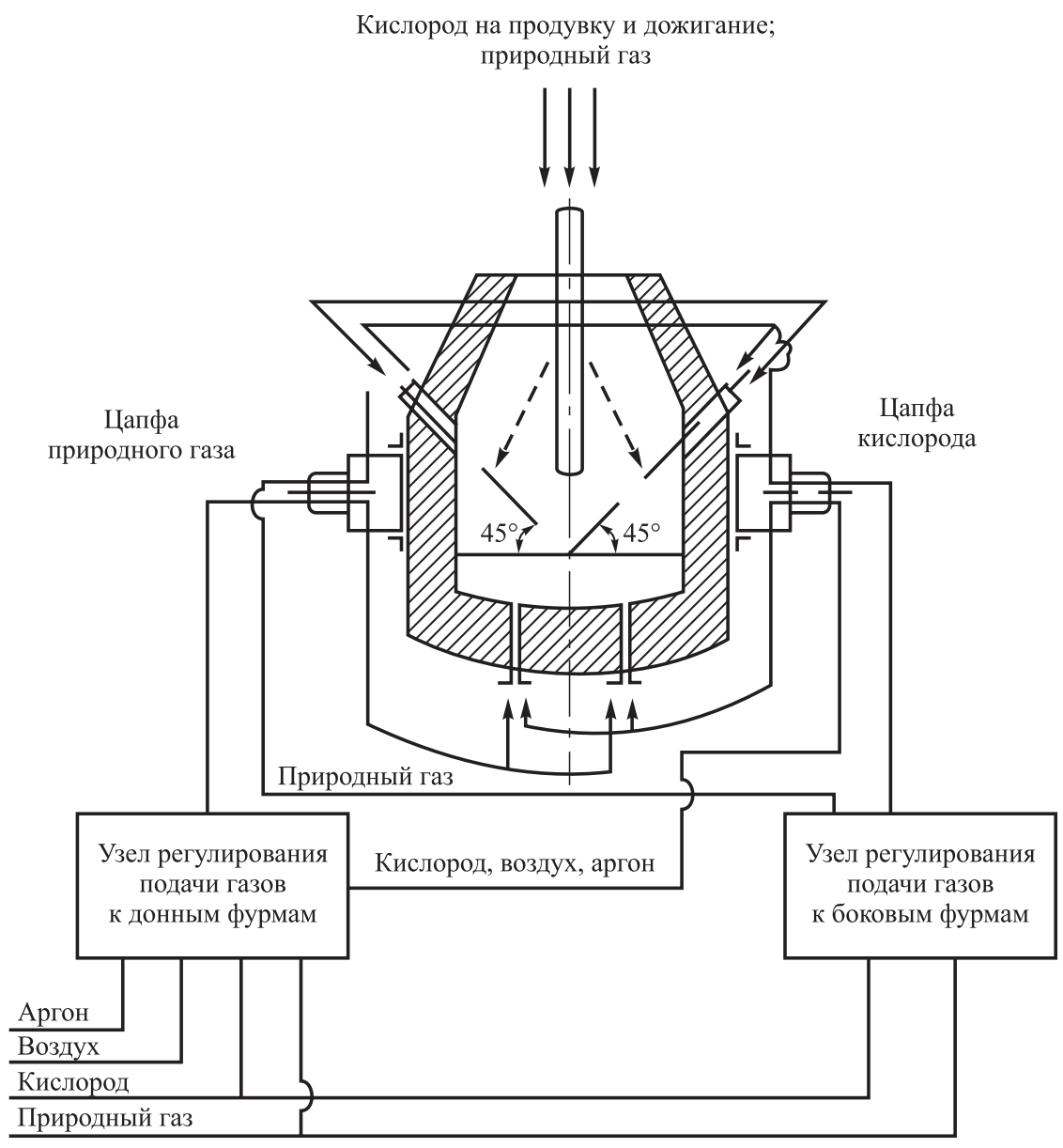

Рис. 1. Схема кислородно-топливного конвертера для выплавки стали

Fig. 1. Scheme of an oxygen-fuel converter for steelmaking 
- разбрызгивание и распыление металла пузырями СО при выходе на поверхность ванны с учетом энергии разрыва пленки пузыря;

- испарение металла в высокотемпературных реакционных зонах.

При определенных допущениях дробление металла на капли за счет динамической энергии струи можно представить как работу образования новой поверхности и выразить как

$$
A=S \sigma,
$$

где $S$ - площадь поверхности раздела фаз, $\mathrm{M}^{2} ; \sigma$ - удельное поверхностное натяжение, Дж/ $\mathrm{M}^{2}$ (например, для железоуглеродистых расплавов в зависимости от содержания углерода при $[\mathrm{C}]=1,0 \div 1,5 \%$ можно принять $\sigma=1250 \cdot 10^{4}$ мДж/ $\mathrm{m}^{2}$ ) [9].

В соответствии с данными работ В.И. Баптизманского и В.Б. Охотского $[1,10]$ на образование новой поверхности (то есть на дробление металла и эжекцию капель в газовые струи) расходуется чуть более 1,0\% динамической энергии струи на выходе из сопла.

Площадь поверхности раздела фаз составляет

$$
S=\frac{A}{\sigma}=\frac{0,01 E_{\mathrm{O}_{2}}}{\sigma},
$$

где $E_{\mathrm{O}_{2}}$ - динамическая энергия струи на выходе из сопла.

Если принять скорость истечения струи из сопел Лаваля порядка $350-500 \mathrm{~m} / \mathrm{c}$, то при расходе $1 \mathrm{~m}^{3}$ кислорода может образоваться поверхность газ-металл - шлак $63-70$ и даже $150-350 \mathrm{~m}^{2}$ при высокой степени дробления расплава на капли [1].

По известному размеру пылевидных частиц можно определить их удельную поверхность [11]:

$$
S_{\text {уд }}=\frac{6}{\rho_{\mathrm{p}}^{M e} d},
$$

где $\rho_{\mathrm{p}}^{M e}-$ плотность распыленного металла; $d$ - диаметр частиц.

При среднем размере пылевидных частиц 10 - 25 мкм их удельная поверхность составит $32-60 \mathrm{~m}^{2} /$ г. Таким образом, 1 м $^{3}$ вдуваемого кислорода за счет распыления металла способен образовать 1,10 - 1,82 г и более пыли.

В результате механического распыления металла газовыми струями выходящие из реакционных зон отходящие газы содержат значительное количество пыли, в особенности в начальный период продувки конвертерной ванны (в условиях отсутствия слоя вспененного шлака) и в период интенсивного газовыделения при высокой скорости окисления углерода. Кроме того, в начале продувки при высоком содержании углерода поверхностное натяжение металла уменьшается, что способствует увеличению вновь образованной поверхности, то есть фактического количества уносимой пыли.

В таких условиях скорость истечения газовых струй, формирующихся в дутьевых устройствах, может значительно превышать звуковую, что резко повышает динамическую энергию струи $[1,3,10,12]$. В результате количество металла, распыленного газовыми струями, может достигать $5-10$ г/м³ отходящих газов, а при «сворачивании» шлака даже $250-350$ г/м ${ }^{3}$ [1]. Бороться с этим источником пылеобразования весьма трудно, так как практически во всех процессах, где применяется продувка ванны, стремятся к увеличению скорости окислительных струй, поскольку это увеличивает степень усвоения кислорода и улучшает перемешивание ванны.

Единственным реальным мероприятием, уменьшающим распыление металла струей, является заглубление ее в ванну. В этом случае образовавшаяся пыль может быть снова поглощена ванной, а значительная часть энергии струй будет использована на образование межфазной поверхности между металлом, шлаком и газом и на создание циркуляционных зон. Примером снижения пылевыделения при заглублении реакционных зон в ванну может служить кислородно-конвертерный процесс с донным дутьем [13 - 15].

При продувке ванны факелом разбрызгивание металла сильнее, чем при кислородной продувке вследствие вдувания большего объема газов. Возможным известным решением проблемы снижения брызгообразования может быть дополнительное перемешивание ванны при использовании механического вращения продувочных устройств или самого агрегата [1 - 4, 13 - 15]. В этом случае объем реакционной зоны увеличивается в 3 - 5 раз по сравнению с обычной продувкой, а энергия струи расходуется не на разбрызгивание расплава, а на дополнительное образование межфазной поверхности.

Представляет интерес оценка процесса распыления металла за счет всплывающих пузырей оксида углерода $\{\mathrm{CO}\}$, который определяется прежде всего скоростью их подъема на поверхность ванны. Так, по данным В.И. Явойского [9] скорость всплывания пузырей $U$ может быть определена по зависимости

$$
U=U_{0}+\frac{m_{\mathrm{O}_{2}}^{2} \omega_{\mathrm{O}_{2}}^{2}}{2 m g}+\frac{V_{\mathrm{CO}} \rho h}{m},
$$

где $U_{0}$ - конвективная скорость металла, вызванная разностью удельных масс (эта скорость имеет малую величину и ею можно пренебречь); $m_{\mathrm{O}_{2}}$ - масса вдуваемого кислорода, кг/c; $\omega_{\mathrm{O}_{2}}-$ скорость истечения кислорода из сопла, м/c; $m$ - масса металла, вовлекаемого струей в циркуляцию, кг; $V_{\mathrm{CO}}-$ скорость образования $\mathrm{CO}, \mathrm{M}^{3} / \mathrm{c}$;

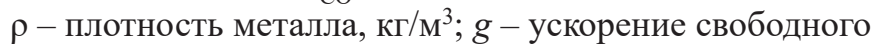
падения, м/ $\mathrm{c}^{2} ; h$ - высота ванны в спокойном состоянии, м. 
Расчеты показывают, что для различных вариантов конвертерного процесса величина $U$ не будет превышать $5 \mathrm{~m} /$. Отсюда следует, что энергия, развиваемая пузырями при всплывании, в сотни раз меньше энергии струи вдуваемого кислорода. Следовательно, можно предположить, что пузыри оксида углерода $\{\mathrm{CO}\}$ образуют во столько же раз меньше пыли.

Следует обратить внимание на то, что пузырь по мере всплывания под действием сил сопротивления приобретает «грибовидную» форму, а скорость всплывания пузыря такой формы определяется по эмпирическому уравнению:

$$
U=\frac{R^{0,4} \sigma_{M e}}{k \rho_{M e}^{0,056} \eta_{M e}^{0,5}}
$$

где $R$ - максимальный радиус пузыря, м; $k$ - эмпирическая постоянная; $\rho_{M e}, \sigma_{M e}$ и $\eta_{M e}-$ плотность, поверхностное натяжение и динамическая вязкость металла.

На интенсивное образование пыли при разрушении оболочек всплывающих пузырей также указывает ряд исследователей $[16,17]$. К основным доказательствам можно отнести известный факт повышения пылеобразования при увеличении скорости обезуглероживания расплава и, в целом, зависимости количества образовавшейся пыли от содержания углерода в ванне. При этом энергия разрыва пузырей зависит от парциального давления газа внутри них и в соответствии с данными работы [18] в начальный момент при зарождении пузырька может составлять $10^{7} \mathrm{H} / \mathrm{M}^{2}$. В процессе всплывания пузыря к поверхности ванны и, соответственно, его росте давление в нем снижается и определяется давлением окружающей среды и поверхностным натяжением жидкой фазы. В соответствии с работами $[9,19]$ величина поверхностного натяжения между газом и шлаком, например, для системы $\mathrm{FeO}-\mathrm{CaO}-\mathrm{SiO}_{2}$ находится в диапазоне $500-600 \mathrm{MH} / \mathrm{m}^{2}$; для металла поверхностное натяжение значительно больше, для расплава с содержанием [C] $\approx 1 \%$ может составлять $1250 \mathrm{MH} / \mathrm{M}^{2}$. Таким образом, пузыри $\{\mathrm{CO}\}$ при выходе на поверхность ванны всегда окружены пленкой металла; такие пузыри называют «корольками». Количество вынесенного металла в данном случае в виде корольков зависит от суммарной величины поверхности пузырей $\{\mathrm{CO}\}$ и может достигать $8-11 \%$ от массы шлака $[1,20,21]$. При этом металлическая пыль, выносимая из зоны продувки, содержит до 60 - 65 \% чистого железа.

Образовавшиеся при разрыве пузыря частицы пыли могут выноситься отходящими газами либо обратно оседать в ванну. Интенсивность их возврата с достаточной степенью точности можно определить в соответствии с теорией кипящего слоя [22]. При всех прочих равных условиях эта интенсивность пропорциональна разности плотностей частицы и отходящего газа

$$
\xi \equiv \sqrt{\rho_{\mathrm{M}}-\rho_{\text {oTx }}},
$$

где $\xi$ - интенсивность возврата брызг в расплав; $\rho_{\text {м }}$ и $\rho_{\text {отх }}$ - плотность выносимой частицы и отходящего газа.

При этом масса частицы зависит от того, окислена она или нет, что, в свою очередь, определяется составом газовой фазы над расплавом. При достаточно высоких температуре и окислительном потенциале газовой фазы, очевидно, протекают реакции окисления частиц с дополнительным пылевыносом.

В соответствии с зависимостью (6) нежелательно допускать окисления распыленных частиц металла: это будет в дальнейшем препятствовать оседанию их в ванну и приводить к сокращению выноса пыли. Для достижения этой цели необходимо стремиться уменьшать окислительный потенциал среды, в которой разрываются пузыри: реализовать можно, например, при разбавлении дутья различными видами топлива или инертным газом (азотом, аргоном). Ранее полученная информация [23] по продувке металла кислородом при разбавлении азотом показывает, что этот метод снижает пылевыделение пропорционально количеству введенного азота для разбавления газового потока. Однако такие методы продувки не получили широкого распространения, так как при этом уменьшаются скорости окислительных реакций, снижается температура ванны [8].

В таком случае более приемлемым способом продувки является добавка топлива к кислороду, которая наряду с уменьшением пылевыделения дополнительно вносит некоторое количество тепла в ванну. Выполненные расчеты условий равновесия между металлом и газовой фазой, состоящей из стехиометрической смеси кислорода и метана, показывают, что газы, выходящие из реакционной зоны, содержат значительное количество $\mathrm{CO}$ и $\mathrm{H}_{2}$, в присутствии которых образование оксидов железа невозможно. Поэтому очевидно, что дисперсные частицы металла в этих условиях будут в большей степени оседать в ванну и меньше выноситься отходящими газами.

Добавка топлива к кислороду уменьшает скорость обезуглероживания, что также способствует уменьшению количества пыли, образующейся при разрыве и дроблении пленок металла газовыми пузырями.

Значительное влияние на температуру реакционной зоны оказывает испарение образующихся оксидов железа и оксидов марганца. При этом расход тепла, связанный с испарением, ограничивает и тормозит значительное повышение температуры реакционной зоны, а предельные значения температуры, очевидно, определяются условиями кипения железа $\left(2735^{\circ} \mathrm{C}\right)$. Многочисленными экспериментальными исследованиями установлено $[1,10,24]$, что при продувке расплава технически чистым кислородом температура в области зоны продувки составляет $2100-2700^{\circ} \mathrm{C}$, а перегрев по отношению к объему продуваемой ванны составляет $700-900{ }^{\circ} \mathrm{C}$. Такой перегрев зависит от содержания 
углерода в металле, скорости обезуглероживания, интенсивности продувки и поэтому изменяется по ходу конвертерной плавки.

При учете фактора испарения железа в реакционной зоне необходимо учитывать, что с повышением температуры упругость паров железа растет по экспоненциальному закону в соответствии с уравнением [1]

$$
\lg P_{\mathrm{Fe}}=\frac{18500}{T}+8,025
$$

здесь $P_{\mathrm{Fe}}-$ упругость паров железа, мм рт. ст.

Выполненные по аналогии с работами $[9,19]$ расчеты показывают, что при таком перегреве реакционной зоны величина $P_{\mathrm{Fe}}$ не превышает 0,04 атм, образуется $4-8$ г/м ${ }^{3}$ пыли; то есть испарение приводит к значительному дополнительному пылевыносу.

Одновременно можно констатировать, что этот источник пылеобразования по своему значению близок к потерям за счет динамического воздействия струи и действию разрывающихся газовых пузырей, то есть не является, как это часто полагают, доминирующим. Этим можно объяснить и тот факт, что попытки устранить дымообразование вдуванием в реакционную зону охладителей хотя и уменьшают его значительно, но кардинально проблему не решают $[2,16,21]$. Поэтому очевидно, что испарение в реакционной зоне не играет определяющей роли в процессе пылеобразования при таких вариантах продувки конвертерной ванны. В качестве подтверждения можно отметить известный факт уменьшения количества пыли в отходящих газах кислородных конвертеров на заключительном этапе продувки (в конце плавки), несмотря на то, что температура ванны в этот период значительно выше, чем в начале процесса. В то же время необходимо отметить, что испарение все же играет определенную роль в пылеобразовании, поэтому использование различных вариантов охлаждения реакционной зоны снижает дымовыделение и пылевынос при выплавке стали.

Надежным и достаточно легко реализуемым способом для снижения пылевыноса от испарения железа в реакционной зоне является добавка газообразного или жидкого топлива к кислородному потоку [1 - 3]. Такой вариант продувки одновременно уменьшает влияние и многих других негативных факторов, вызывающих образование пыли. В данном случае реализация совместно с кислородно-топливной продувкой в конвертере позволяет параллельно увеличить приход тепла в ванну.

Для оценки влияния изменения температуры $(\Delta T)$ реакционной зоны при продувке ванны высокотемпературным метанокислородным факелом использовали сопоставляемые варианты технологии продувки газокислородной смесью на примере углеродистой и легированной стали для прокатных валков 7Х2СМ2Ф.

В такой постановке задачи температура металла на условной границе реакционной зоны зависит от коли- чества тепла $Q$, переданного металлу из зоны продувки, и размеров образующейся реакционной зоны.

Процесс отвода тепла из реакционной зоны в объем ванны можно описать уравнением Ньютона

$$
Q=\alpha\left(T_{\mathrm{p} .3}-T_{M e}\right) F_{\mathrm{p} .3},
$$

где $\alpha$ - коэффициент теплоотдачи (зависит от скорости циркуляции металла и его теплофизических свойств (вязкости, теплопроводности, удельной теплоемкости и т.д.); $T_{\text {p.з }}$ и $T_{M e}-$ температура реакционной зоны и металла, К; $F_{\text {p.3 }}$ - площадь реакционной зоны.

Для расчета температуры реакционной зоны с учетом гидродинамических и тепловых явлений у границ зоны воспользовались теорией теплового пограничного слоя (законом Фурье).

При допущении, что для обоих вариантов технологии продувки углеродистой и легированной стали коэффициенты теплоотдачи $\alpha$ равны, то уравнением (8) можно воспользоваться для сравнения основных тепловых процессов в реакционной зоне. Тогда удельный тепловой поток $q$ из реакционной зоны в объем конвертерной ванны можно определить по зависимости

$$
q=\frac{Q}{F_{\mathrm{p} .3}}=\alpha \Delta T \text { или } \Delta T=\frac{Q}{\alpha F_{\mathrm{p} .3}},
$$

где $\Delta T=T_{\mathrm{p} .3}-T_{M e}$.

Приняв $\alpha_{1}=\alpha_{2}$, можно записать

$$
\frac{\Delta T_{\text {л }}}{\Delta T_{\mathrm{y}}}=\frac{Q_{\mathrm{I}} F_{\mathrm{p} \cdot 3_{1}}}{Q_{\mathrm{y}} F_{\mathrm{p} .3_{2}}},
$$

где $\Delta T_{\text {л }}$ и $\Delta T_{\text {у }}$ - перегрев температуры реакционной зоны над усредненной в ванне для легированной и углеродистой сталей; $Q_{\text {л }}$ и $Q_{\text {у }}$ - тепловой эффект реакции окисления элементов ванны продуктами горения метана при продувке легированной и углеродистой стали; $F_{\text {p.31 }}$ и $F_{\text {p.32 }}-$ площадь реакционной зоны при производстве легированной (индекс 1) и углеродистой (индекс 2) стали.

В соответствии с принятым допущением $F_{\text {p.31 }}=F_{\text {p.32 }}$ выражение (10) можно упростить

$$
\frac{\Delta T_{\text {л }}}{\Delta T_{\mathrm{y}}}=\frac{Q_{\text {Л }}}{Q_{\mathrm{y}}} .
$$

С точки зрения решения задачи уменьшения испарения металла в реакционной зоне выгодным является режим, при котором отношение $\frac{Q}{F_{\text {p.3 }}}$ наименьшее.

В соответствии с законом действующих масс в реакционной зоне происходит преимущественное окисление железа. Пренебрегая диссоциацией молекул на 
ионы и атомы, для упрощения расчетов можно принять, что в реакционной зоне одновременно протекают:

- окисление метана кислородом

$$
\mathrm{O}_{2}+m \mathrm{CH}_{4}=m \mathrm{CO}_{2}+2 m \mathrm{H}_{2} \mathrm{O}+(1-2 m) \mathrm{O}_{2} \text {; }
$$

- окисление металла кислородом и продуктами горения $\left(\mathrm{CO}_{2}\right.$ и $\left.\mathrm{H}_{2} \mathrm{O}\right)$

$$
\begin{gathered}
M e+m \mathrm{CO}_{2}+2 m \mathrm{H}_{2} \mathrm{O}+(1-2 m) \mathrm{O}_{2}= \\
=\left(1-\mathfrak{x}_{\mathrm{CO}_{2}}\right) m \mathrm{CO}_{2}+2\left(1-\mathfrak{\mathrm { H }}_{2} \mathrm{O}\right) m \mathrm{H}_{2} \mathrm{O}+ \\
+\mathfrak{}_{\mathrm{CO}_{2}} m \mathrm{CO}+2 \mathfrak{x}_{\mathrm{H}_{2} \mathrm{O}} m \mathrm{H}_{2}+\left[\mathfrak{C}_{\mathrm{CO}_{2}} m+2 \mathfrak{\mathrm { H }}_{2} \mathrm{O}\right. \\
+n(1-2 m)] M e \mathrm{O}
\end{gathered}
$$

где $m$ - количество молей метана $\mathrm{CH}_{4}$, приходящихся на 1 моль $\mathrm{O}_{2} ; æ_{\mathrm{CO}_{2}}$ и $\mathfrak{H}_{\mathrm{H}_{2} \mathrm{O}}$ - степень химического взаимодействия $\mathrm{CO}_{2}$ и $\mathrm{H}_{2} \mathrm{O}$ с элементами расплава; $n$ - множитель, уравнивающий валентности соответствующего элемента и окислителя в правой и левой частях уравнения.

Величина $m$ связана обратной зависимостью с коэффициентом расхода кислорода $\Lambda$ в потоке. Тогда при добавке топлива $(m=0 \div 0,5)$ продуктами горения являются $\mathrm{CO}_{2}$ и $\mathrm{H}_{2} \mathrm{O}$ и избыточный кислород. При $m>0,5$ в продуктах горения, кроме упомянутых компонентов, присутствуют $\mathrm{CO}$ и $\mathrm{H}_{2}$. При окислительной продувке рассмотрение тепловых эффектов реакций проводили только при $m=0 \div 0,5$;

Реакции окисления железа продуктами горения и константы равновесия $\left(K_{\mathrm{p}}\right)$ этих реакций можно записать

$$
\begin{aligned}
& {[\mathrm{Fe}]+\left[\mathrm{CO}_{2}\right]=(\mathrm{FeO})+(\mathrm{CO}), K_{\mathrm{p}}^{\mathrm{a}}=\frac{P_{\mathrm{CO}} C_{\mathrm{FeO}}}{P_{\mathrm{CO}_{2}}} ;} \\
& {[\mathrm{Fe}]+\left[\mathrm{H}_{2} \mathrm{O}\right]=(\mathrm{FeO})+\left(\mathrm{H}_{2}\right), K_{\mathrm{p}}^{\sigma}=\frac{P_{\mathrm{H}_{2}} C_{\mathrm{FeO}}}{P_{\mathrm{H}_{2} \mathrm{O}}},}
\end{aligned}
$$

где $P$ - парциальное давление компонентов; $C$ - концентрация компонентов.

Тогда завершенность протекания реакций (14) и (15) (то есть использование кислорода продуктов горения на окисление металла) будет равно $(1-æ)$, соответственHо, $P_{\mathrm{CO}}=æ P ; P_{\mathrm{CO}_{2}}=(1-æ) P ; P_{\mathrm{H}_{2}}=æ P ; P_{\mathrm{H}_{2} \mathrm{O}}=(1-\mathfrak{x}) P$.

При стандартных условиях $(P=1$ атм $)$ и $\mathrm{FeO} \leq 1 \%$ константы равновесия рассматриваемых реакций $K_{\mathrm{p}}^{\mathrm{a}, \sigma}=\frac{\mathfrak{x}}{1-\mathfrak{x}}$, откуда $\mathfrak{x}=\frac{K_{\mathrm{p}}^{\mathrm{a}, \sigma}}{1+K_{\mathrm{p}}^{\mathrm{a}, \sigma}}, \lg K_{\mathrm{p}}^{\mathrm{a}, \sigma}=-\frac{\Delta z_{\mathrm{a}, \sigma}^{\mathrm{o}}}{4,575 T}($ где $\Delta Z_{\mathrm{a}, \text { б }}$ - изменение изобарно-изотермического потенциала реакций окисления железа продуктами горения). Эта величина для реакций (14) и (15) легко определяется с помощью табличных данных [25]. В зависимости от температуры изменение степени химического взаимодействия продуктов горения с углеродистой сталью представлено на рис. 2 (кривые 1 и $l^{\prime}$ ). В интервале температур 1800 - 2500 К средняя степень химического взаимодействия продуктов горения метана при продувке в погружном режиме составляет $60-75 \%$.

Добавка метана к кислородному дутью способна связывать в реакционной зоне большое количество кислорода, снижая в целом окислительную способность дутья. Это должно уменьшить угар металла по сравнению с продувкой чистым кислородом. Следовательно, степень разложения в ванне $\mathrm{CO}_{2}$ и $\mathrm{H}_{2} \mathrm{O}$ является одной из основных характеристик газо-кислородного способа продувки, определяющих окислительные и нагревательные свойства дутья.

При продувке стали для прокатных валков (содержащей более $90 \% \mathrm{Fe}$, примерно $2 \%$ Сr и $2 \% \mathrm{Mo}$ ) реакции окисления этих элементов продуктами окисления метана и избыточным кислородом следующие:

$$
\begin{gathered}
2 \mathrm{Fe}+m \mathrm{CO}_{2}+2 m \mathrm{H}_{2} \mathrm{O}(1-2 m) \mathrm{O}_{2}= \\
=\left(1-\mathfrak{C O}_{\mathrm{CO}_{2}}\right) m \mathrm{CO}_{2}+2\left(1-\mathfrak{H}_{\mathrm{H}_{2} \mathrm{O}}\right) m \mathrm{H}_{2} \mathrm{O}+ \\
+\mathfrak{x}_{\mathrm{CO}_{2}} m \mathrm{CO}+2 \mathfrak{x}_{\mathrm{H}_{2} \mathrm{O}} m \mathrm{H}_{2}+ \\
+\left[\mathfrak{x}_{\mathrm{CO}_{2}} m+2 \mathfrak{x}_{\mathrm{H}_{2} \mathrm{O}} m+2(1-2 m)\right] \mathrm{FeO} ;
\end{gathered}
$$

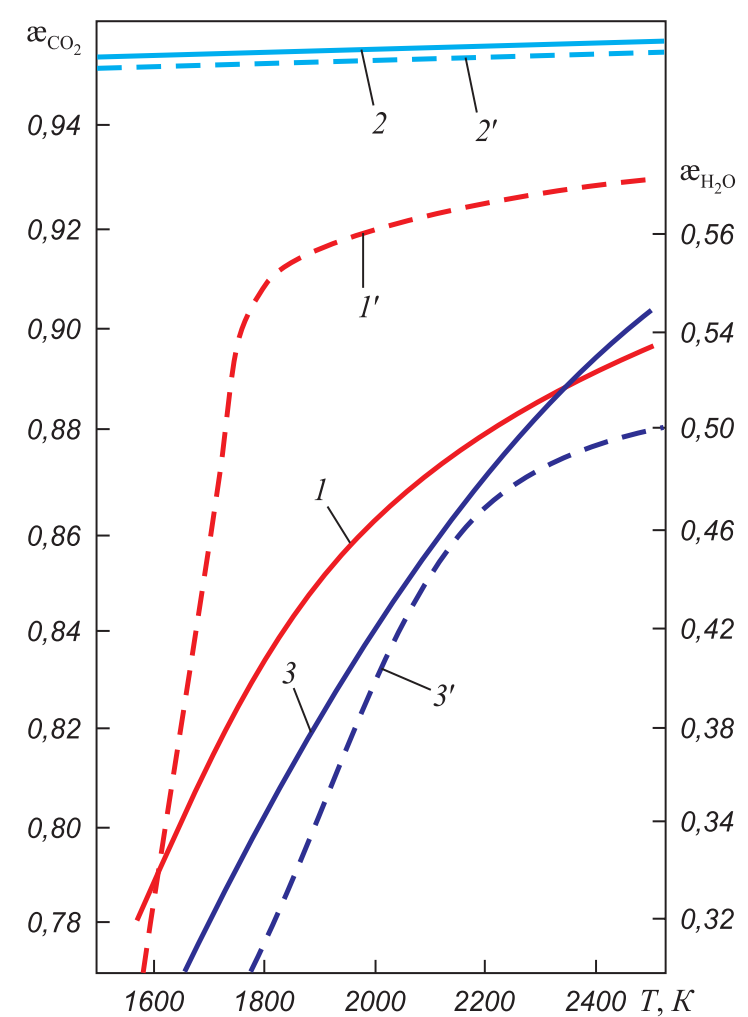

Рис. 2. Зависимость степени взаимодействия продуктов горения метана от температуры реакционной зоны при взаимодействии с элементами расплава углеродистой стали:

$-\mathrm{CO}_{2} ;----\mathrm{H}_{2} \mathrm{O} ; 1,1^{\prime}-\mathrm{Fe} ; 2,2^{\prime}-\mathrm{Cr} ; 3,3^{\prime}-\mathrm{Mo}$

Fig. 2. Dependence of the degree of methane combustion products interaction on temperature of the reaction zone when interacting with the carbon steel melt elements:

$-\mathrm{CO}_{2} ;----\mathrm{H}_{2} \mathrm{O} ; 1,1^{\prime}-\mathrm{Fe} ; 2,2^{\prime}-\mathrm{Cr} ; 3,3^{\prime}-\mathrm{Mo}$ 


$$
\begin{gathered}
\frac{2}{3} \mathrm{Cr}+m \mathrm{CO}_{2}+2 m \mathrm{H}_{2} \mathrm{O}(1-2 m) \mathrm{O}_{2}= \\
=\left(1-\mathfrak{x}_{\mathrm{CO}_{2}}\right) m \mathrm{CO}_{2}+2\left(1-\mathfrak{x}_{\mathrm{H}_{2} \mathrm{O}}\right) m \mathrm{H}_{2} \mathrm{O}+ \\
+\mathfrak{x}_{\mathrm{CO}_{2}} m \mathrm{CO}+2 \mathfrak{x}_{\mathrm{H}_{2} \mathrm{O}} m \mathrm{H}_{2}+ \\
+\left[\mathfrak{x}_{\mathrm{CO}_{2}} m+2 \mathfrak{x}_{\mathrm{H}_{2} \mathrm{O}} m+\frac{2}{3}(1-2 m)\right] \mathrm{Cr}_{2} \mathrm{O}_{3} ; \\
\quad \mathrm{Mo}+m \mathrm{CO}_{2}+2 m \mathrm{H}_{2} \mathrm{O}(1-2 m) \mathrm{O}_{2}= \\
=\left(1-\mathfrak{x}_{\mathrm{CO}_{2}}\right) m \mathrm{CO}_{2}+2\left(1-\mathfrak{x}_{\mathrm{H}_{2} \mathrm{O}}\right) m \mathrm{H}_{2} \mathrm{O}+ \\
\quad+\mathfrak{}_{\mathrm{CO}_{2}} m \mathrm{CO}+2 \mathfrak{x}_{\mathrm{H}_{2} \mathrm{O}} m \mathrm{H}_{2}+ \\
+\left[\mathfrak{x}_{\mathrm{CO}_{2}} m+2 \mathfrak{x}_{\mathrm{H}_{2} \mathrm{O}} m+2(1-2 m)\right] \mathrm{MoO}_{2} .
\end{gathered}
$$

Тепловой эффект реакций (12) и (13) составит

$$
\begin{aligned}
Q= & {\left[\mathfrak{}_{\mathrm{CO}_{2}} m+2 \mathfrak{x}_{\mathrm{H}_{2} \mathrm{O}} m+n(1-2 m)\right] Q_{\mathrm{MeO}}+} \\
+ & m\left(1-\mathfrak{x}_{\mathrm{CO}_{2}}\right) Q_{\mathrm{CO}_{2}}+\mathfrak{C O}_{\mathrm{CO}_{2}} m Q_{\mathrm{CO}_{2}}+ \\
& +2\left(1-\mathfrak{x}_{\mathrm{H}_{2} \mathrm{O}}\right) m Q_{\mathrm{H}_{2} \mathrm{O}}-m Q_{\mathrm{CH}_{4}} .
\end{aligned}
$$

В целом при определенных допущениях с учетом концентраций суммарный тепловой эффект реакции окисления легированной стали составит

$$
Q_{\mathrm{r}}=0,9 Q_{16}+0,02 Q_{17}+0,02 Q_{18}-m Q_{\mathrm{CH}_{4}},
$$

где $Q_{16}, Q_{17}, Q_{18}$-тепловые эффекты реакций (16) - (18).

Расчет теплового эффекта сделан во всем диапазоне изменений æ. При $T_{M e}=1800$ К теплота образования оксидов $\mathrm{FeO}, \mathrm{Cr}_{2} \mathrm{O}_{3}, \mathrm{CO}_{2}, \mathrm{CO}, \mathrm{H}_{2} \mathrm{O}, \mathrm{MoO}_{2}$ и $\mathrm{CH}_{4}$, отнесенная к одному молю, принята 54 700, 270 500, 94 100, 28 080, 60 110, 130800 и 22100 ккал/моль $\mathrm{O}_{2}$.

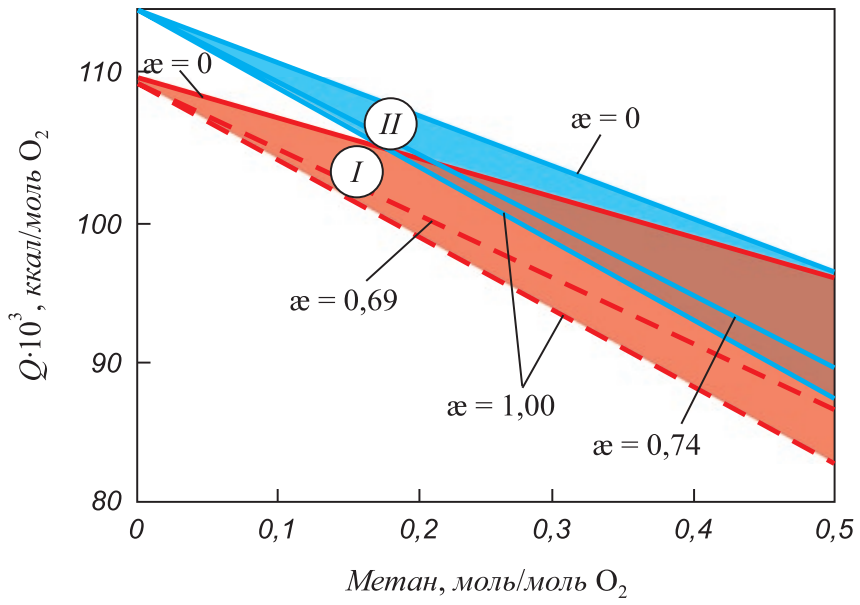

Рис. 3. Зависимость количества тепла, выделяющегося в реакционной зоне, от доли метана в кислородной струе при продувке углеродистой (зона $I$ ) и легированной (зона $I I$ ) сталей при различных значениях æ

Fig. 3. Dependence of the amount of heat released in the reaction zone on proportion of methane in the oxygen jet during carbon $(I)$ and alloyed $(I I)$ steels blasting at different values of $æ$
Результаты расчетов по уравнениям (19) и (20) представлены на рис. 3. По мере увеличения степени разбавления кислорода природным газом и, соответственно, увеличения степени разложения продуктов горения количество тепла, передаваемого от зоны продувки в объем металла, уменьшается в обоих случаях, причем при продувке легированной стали процесс идет более интенсивно. Это свидетельствует о том, что реакции образования оксидов хрома, молибдена и других элементов более эндотермичные, чем окисление железа до $\mathrm{FeO}$. Кроме того, при продувке углеродистой стали при $1800 \mathrm{~K}$ среднее значение степени разложения продуктов горения $æ_{\text {ср }}$ составляет 0,69 (рис. 3), в то время как для легированной стали прежде всего из-за наличия хрома оно смещается ближе к единице $\left(æ_{c p}=0,74\right)$, что, в свою очередь, также способствует уменьшению количества тепла, выделяющегося в реакционной зоне. Изменение тепловых эффектов реакций окисления элементов ванны при $\mathfrak{c p}_{\text {ср }}$ также показано на рис. 3.

Чтобы по уравнению (11) оценить, как влияет добавка природного газа на величину $\Delta T_{\text {л }}$, необходимо знать значения $Q_{\mathrm{y}}$ и $\Delta T_{\mathrm{y}}$, для определения которых можно использовать данные работы [25] (рис. 4, кривая 1).

Если конкретные значения $\Delta T_{\mathrm{y}}, Q_{\mathrm{y}}, Q_{\text {л }}$ (рис. 3,4 ) подставить в уравнение (11), то можно вычислить значение $\Delta T_{\text {л }}$. Принимая температуру ванны $1800 \mathrm{~K}$ и суммируя с ней $\Delta T_{\text {л }}$, можно определить теоретическую температуру в реакционной зоне и влияние на нее добавки метана при продувке легированной стали погружным газокислородным факелом (рис. 4, кривая 2). По мере увеличения доли метана температура в реакционной зоне снижается, и, соответственно, уменьшается испарение железа в ней. Как установлено, оптимальные условия продувки легированной стали газокислородным факелом достигаются при коэффициенте расхода кислорода $\Lambda=1,8 \div 2,0$ $(m \leq 0,278)$. В этом случае температура в реакционной зоне (по сравнению с продувкой чистым кислородом)

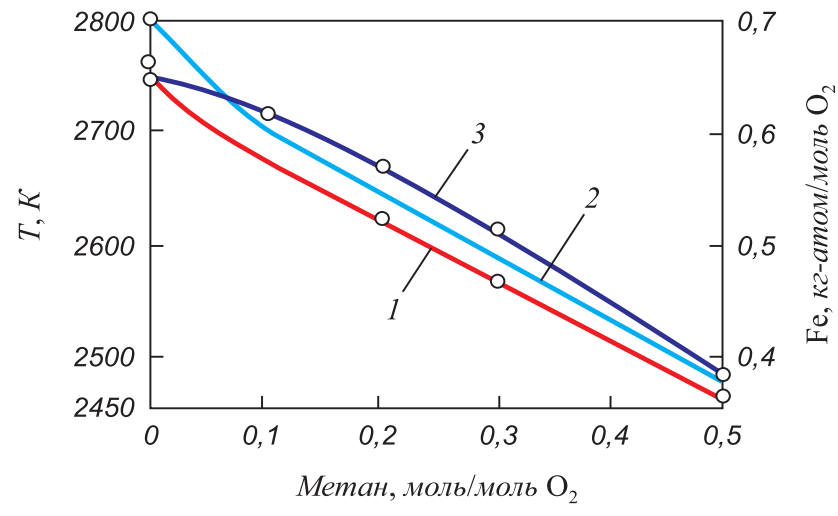

Рис. 4. Влияние добавки метана к кислородному потоку на температуру в реакционной зоне углеродистой стали (1), легированной стали (2) и на испарение железа (3)

Fig. 4. Influence of methane introduction to the oxygen flow on temperature in reaction zone of carbon steel (1), alloyed steel (2) and on iron evaporation (3) 
снижается на $200-210^{\circ} \mathrm{C}$, что также уменьшает испарение железа в 1,3 раза (рис. 4, кривая 3 ).

Уменьшение количества тепла, поступающего в объем ванны из реакционной зоны, при увеличении доли разбавления кислорода природным газом не обязательно приводит к снижению скорости нагрева ванны в целом. Объясняется это тем, что взаимодействие металла с продуктами полного горения газа $\left(\mathrm{CO}_{2}\right.$ и $\left.\mathrm{H}_{2} \mathrm{O}\right)$ приводит к выделению в ванне дополнительного тепла, величину которого можно оценить с помощью теплового эффекта реакции окисления углерода продуктами горения и избыточным кислородом:

$$
\begin{gathered}
m \mathrm{CH}_{4}+\mathrm{O}_{2}\left[2(1-2 m)+\mathfrak{x}_{\mathrm{CO}_{2}} m+2 \mathfrak{x}_{\mathrm{H}_{2} \mathrm{O}} m\right][\mathrm{C}]= \\
=\left[2 \mathfrak{}_{\mathrm{H}_{2} \mathrm{O}} m+2 \mathfrak{C}_{\mathrm{CO}_{2}} m+2(1-2 m)\right] \mathrm{CO}_{2}+ \\
+\left(1-\mathfrak{x}_{\mathrm{CO}_{2}}\right) m \mathrm{CO}_{2}+2\left(1-\mathfrak{x}_{\mathrm{H}_{2} \mathrm{O}}\right) m \mathrm{H}_{2} \mathrm{O}+ \\
+2 \mathfrak{x}_{\mathrm{H}_{2} \mathrm{O}} m \mathrm{H}_{2} .
\end{gathered}
$$

Тепловой эффект реакции (21), рассчитанный на основании закона Гесса при допущениях, что $\mathfrak{C O}_{2}=\mathfrak{2}_{\mathrm{H}_{2} \mathrm{O}}=$ $=æ$, а температура конечных продуктов реакции равна температуре ванны $\left(T_{M e}=1800 \mathrm{~K}\right)$, составит

$$
Q_{\Sigma}=56160+84070 m-92070 æ m .
$$

Тогда количество тепла, усвоенного ванной, определяется как разность между суммарным теплом $Q_{\Sigma}$ и теплом, теряемым с отходящими из агрегата газами

$$
Q_{\text {усв }}-Q_{\Sigma}-Q_{\text {отх }},
$$

где $Q_{\text {отх }}=V_{\Gamma} C_{\Gamma} T_{M e} ; C_{\text {г }}=C_{\Sigma}=\sum c_{j} n_{j}-$ удельная теплоемкость конвертерного газа, представляющего собой смесь нескольких газов; $c_{j}$ - удельная теплоемкость каждого газа в смеси, вычисленная при температуре $T_{\text {отх.г }} ; n$ - число компонентов газа в смеси.

При определении количества тепла $Q_{\text {отх }}$, теряемого с отходящими газами, объем отходящих газов $V_{\text {отх }}$ рассчитывается, а их теплоемкость $C_{\text {г }}$ может быть принята в соответствии с данными работы [25]. Как видно (рис. 5), количество тепла, усвоенного ванной от реакции окисления углерода $Q_{\text {y }}$, при малых значениях степени разложения продуктов горения $\left(\mathfrak{c}_{\mathrm{cp}}\right.$ менее 0,6$)$ возрастает по мере увеличения доли природного газа в кислородном потоке, что подтверждают и другие экспериментальные данные [26]. Значению æ $>0,7$ соответствует меньшая величина $Q_{\mathrm{y}}$, а при $æ=1,0$ и $m>0,45$ ванна даже охлаждается, так как при полной диссоциации продуктов горения реакция окисления углерода будет идти с эндотермическим эффектом. В целом, в соответствии с выражением (21) окислительная способность газокислородного дутья может быть описана выражением

$$
\left[2(1-2 m)+\mathrm{CO}_{2} m+2 \mathrm{H}_{2} \mathrm{O} m\right][\mathrm{C} \%] .
$$

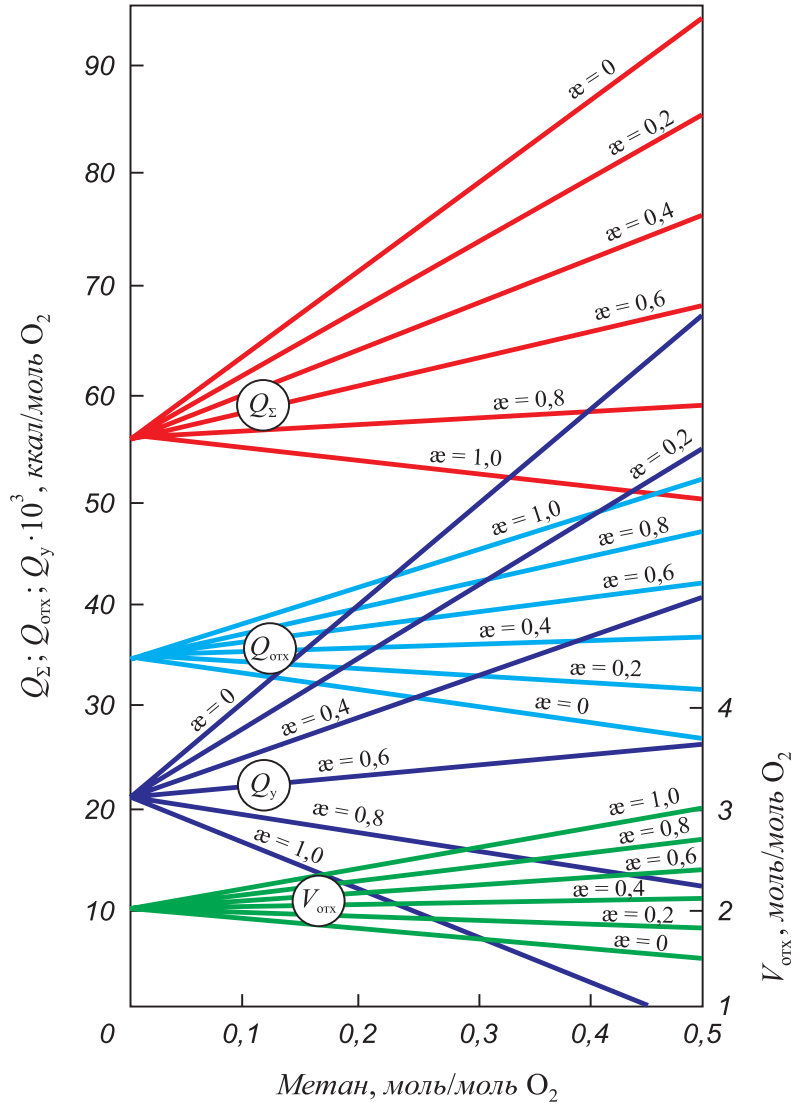

Рис. 5. Изменение суммарного $Q_{\Sigma}$, теряемого $Q_{\text {отх }}$ с отходящими

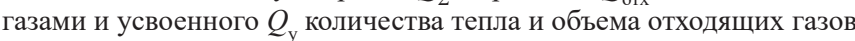
$V_{\text {отх }}$ в зависимости от добавки метана к кислороду при продувке

Fig. 5. Change in total $Q_{\Sigma}$, lost $Q_{\text {отх }}$ with exhaust gases, the amount $Q_{\mathrm{y}}$ of heat consumed and the volume of exhaust gases $V_{\text {отх }}$, depending on methane introduction to oxygen during blasting

Окислительную способность такого факела можно изменять в самом широком диапазоне, при этом решать различные технологические задачи процесса.

\section{Выводы}

Добавка топлива в кислородные струи и реализация газо-кислородной продувки является эффективным способом уменьшения пылеобразования в конвертерной плавке. Такое техническое решение позволяет снижать температуру реакционной зоны и, соответственно, уменьшает количество выделяемого дыма, изменяет характер окисления распыленных капель металла, способствует коагуляции частиц и возвращению их в ванну. Одновременно улучшается тепловой баланс процесса. В то же время добавка топлива к дутью в целом не позволяет снизить пылевыделение до таких пределов, чтобы можно было отказаться от использования эффективных систем газоочистки. Для уменьшения капитальных и эксплуатационных затрат системы газоочистки необходимо снижать содержание пыли в отходящих газах, применяя различные технологические способы уменьшения пылеобразования в процессе продувки. 
1. Баптизманский В.И., Меджибожский М.Я., Охотский В.Б. Конвертерные процессы производства стали. Киев-Донецк: Вища школа, 1984. 343 с.

2. Технология производства стали в современных конвертерных цехах / С.В. Колпаков, Р.В. Старов, В.В. Смоктий и др. М.: Машиностроение, 1991. $464 \mathrm{c}$.

3. Баптизманский М.И., Бойченко Б.М., Черевко В.П. Тепловая работа кислородных конвертеров. М.: Металлургия, 1988. $174 \mathrm{c}$.

4. Li M., Li L., Zhang B., Li Q., Wu W., Zou Z. Numerical analysis of the particle-induced effect on gas flow in a supersonic powder-laden oxygen jet // Metallurgical and Materials Transactions B: Process Metallurgy and Materials Processing Science. 2020. Vol. 51. No. 4. P. 1718-1730. http://doi.org/10.1007/s11663-020-01855-3

5. Ashrit S., Sarkar S., Chatti R.V., Sarkar C., Sarkar S. Nonmetallic LD slag fines - opportunities by invoking chemistry // Ironmaking and Steelmaking. 2020. Vol. 47. No. 8. P. 903-907. http://doi.org/10. 1080/03019233.2019.164167

6. Wang B., Shen S., Ruan Y., Cheng S., Peng W., Zhang J. Simulation of gas-liquid two-phase flow in metallurgical process // Acta Metallurgica Sinica. 2020. Vol. 56. No. 4. P. 619-632. http://doi.org/10. 11900/0412.1961.2019.00385

7. Yao L., Zhu R., Tang Y., Wei G., Dong K. Effect of furnace gas composition on characteristics of supersonic oxygen jets in the converter steelmaking process // Materials. 2020. Vol. 13. No. 15. Article 3353. http://doi.org/10.3390/ma13153353

8. Sharma S.K., Hlinka J.W., Kern D.W. The bath circulation, jet penetration and high-temperature reaction zone in BOF steelmaking // Steelmaking Proceedings. 1977. Vol. 60. P. 181-197.

9. Явойский В.И. Теория процессов производства стали. М.: Металлургия, 1969. 467 с.

10. Охотский В.Б. Физико-химическая механика сталеплавильных процессов. М.: Металлургия, 1993. 151 с.

11. Федорченко И.М., Андриевский С.А. Основы порошковой металлургии. Киев: Изд-во АН УССР, 1963. 420 с

12. Barella S., Mapelli C., Mombelli D., Gruttadauria A., Laghi E., Ancona V., Valentino G. Model for the final decarburisation of the steel bath through a self-bubbling effect // Ironmaking and Steelmaking. 2019. Vol. 46. No. 8. P. 721-724. http://doi.org/10.1080/03019233.2 017.1405179

13. Jacobs H. Q-BOP process - after eleven years // Metals. 1973 Vol. 25. No. 3. P. 33-41

14. Li W., Zhu R., Feng C., Wei G., Han B. Influence of carrier gas of converter oxygen lance on smooth distribution of $\mathrm{O}_{2}-\mathrm{CO}_{2}-\mathrm{CaO}$ mixed jet // Transactions of the Indian Institute of Metals. 2020. Vol. 73. No. 12. P. 3027-3035. http://doi.org/10.1007/s12666-02002105-5

15. Osani H., Ohmiya S. Total hot metals pretreatment and BOF operation practice for high purity steelmaking. In: 1 EOS Congress Düsseldorf, 1993. P. 41-46.

16. Jamamoto Z. Production and technology of iron and steel in Japan during 1993 // ISIJ International. 1994. Vol. 34. No. 4. P. 229-312. https://doi.org/10.2355/isijinternational.34.299

17. Somways N.L. Development in the North-American iron and steel industry // Iron and Steel Engineering. 1994. Vol. 71. No. 2. P. 1-20.

18. Филиппов С.И., Кольцов А.Т. Распределение парциального давления кислорода вдоль многофазного дутьевого потока кислородного конвертера // Известия вузов. Черная металлургия. 1979. № 9. С. 33-36.

19. Меджибожский М.Я. Основы термодинамики и кинетики сталеплавильных процессов. Киев-Донецк: Вища школа, 1979. $277 \mathrm{c}$.

20. Протопопов Е.В., Соломон Г.М., Веревкин Г.И. Состояние шлакометаллической эмульсии и изменения физико-химических характеристик по ходу плавки в кислородном конверторе // Известия вузов. Черная металлургия. 1995. № 8. С. 25-27.
1. Baptizmanskii V.I., Medzhibozhskii M.Ya., Okhotskii V.B. Converter Steelmaking Processes. Kiev-Donetsk: Vishcha shkola, 1984, 343 p. (In Russ.).

2. Kolpakov S.V., Starov R.V., Smoktii V.V. etc. Steelmaking Technology in Modern Converter Plants. Moscow: Mashinostroenie, 1991, 464 p. (In Russ.).

3. Baptizmanskii M.I., Boichenko B.M., Cherevko V.P. Thermal Operation of Oxygen Converters. Moscow: Metallurgiya, 1988, 174 p. (In Russ.).

4. Li M., Li L., Zhang B., Li Q., Wu W., Zou Z. Numerical analysis of the particle-induced effect on gas flow in a supersonic powder-laden oxygen jet. Metallurgical and Materials Transactions B: Process Metallurgy and Materials Processing Science. 2020, vol. 51, no. 4, pp. 1718-1730. http://doi.org/10.1007/s11663-020-01855-3

5. Ashrit S., Sarkar S., Chatti R.V., Sarkar C., Sarkar S. Nonmetallic LD slag fines - opportunities by invoking chemistry. Ironmaking and Steelmaking. 2020, vol. 47, no. 8, pp. 903-907. http://doi.org/10. 1080/03019233.2019.164167

6. Wang B., Shen S., Ruan Y., Cheng S., Peng W., Zhang J. Simulation of gas-liquid two-phase flow in metallurgical process. Acta Metallurgica Sinica. 2020, vol. 56, no. 4, pp. 619-632. http://doi.org /10.11900/0412.1961.2019.00385

7. Yao L., Zhu R., Tang Y., Wei G., Dong K. Effect of furnace gas composition on characteristics of supersonic oxygen jets in the converter steelmaking process. Materials. 2020, vol. 13, no. 15, article 3353. http://doi.org/10.3390/ma13153353

8. Sharma S.K., Hlinka J.W., Kern D.W. The bath circulation, jet penetration and high-temperature reaction zone in BOF steelmaking. Steelmaking Proceedings. 1977, vol. 60, pp. 181-197.

9. Yavoiskii V.I. Theory of Steelmaking Processes. Moscow: Metallurgiya, 1969, 467 p. (In Russ.).

10. Okhotskii V.B. Physicochemical Mechanics of Steelmaking Processes. Moscow: Metallurgiya, 1993, 151 p. (In Russ.).

11. Fedorchenko I.M., Andrievskii S.A. Fundamentals of Powder Metallurgy. Kiev: Izd-vo AN USSR, 1963, 420 p. (In Russ.).

12. Barella S., Mapelli C., Mombelli D., Gruttadauria A., Laghi E., Ancona V., Valentino G. Model for the final decarburisation of the steel bath through a self-bubbling effect. Ironmaking and Steelmaking. 2019, vol. 46, no. 8, pp. 721-724. http://doi.org/10.1080/03019233. 2017.1405179

13. Jacobs H. Q-BOP process - after eleven years. Metals. 1973, vol. 25 , no. 3, pp. 33-41.

14. Li W., Zhu R., Feng C., Wei G., Han B. Influence of Carrier Gas of Converter Oxygen Lance on Smooth Distribution of $\mathrm{O}_{2}-\mathrm{CO}_{2}-\mathrm{CaO}$ Mixed Jet. Transactions of the Indian Institute of Metals. 2020, vol. 73, no. 12, pp. 3027-3035. http://doi.org/10.1007/s12666-02002105-5

15. Osani H., Ohmiya S. Total hot metals pretreatment and BOF operation practice for high purity steelmaking. In: 1 EOS Congress. Düsseldorf, 1993, pp. 41-46.

16. Jamamoto Z. Production and technology of iron and steel in Japan during 1993. ISIJ International. 1994, vol. 34, no. 4, pp. 299-312. https://doi.org/10.2355/isijinternational.34.299

17. Somways N.L. Development in the North-American iron and steel industry. Iron and Steel Engineering. 1994, vol. 71, no. 2, pp. 1-20.

18. Filippov S.I., Kol'tsov A.T. Oxygen partial pressure distribution along a multiphase blast stream of oxygen converter. Izvestiya. Ferrous Metallurgy. 1979, no. 9, pp. 33-36. (In Russ.).

19. Medzhibozhskii M.Ya. Fundamentals of Thermodynamics and Kinetics of Steelmaking Processes. Kiev-Donetsk: Vishcha shkola, 1979, 277 p. (In Russ.).

20. Protopopov E.V., Solomon G.M., Verevkin G.I. State of slag-metal emulsion and changes in physicochemical properties during melting in an oxygen converter. Izvestiya. Ferrous Metallurgy. 1995, no. 8, pp. 25-27. (In Russ.) 
21. Goodman N. Slag splashing of BOF converters // Iron and Steel Inst. 1996. No. XXX. P. 24-33.

22. Diepmann D., Gharib M. The role of streamwise vortisity in the hear-field entrainment of round jets // Journal of Fluid Mechanics. 1992. Vol. 245. P. 643-668.

23. Протопопов Е.В., Айзатулов Р.С., Чернятевич А.Г. Технологические аспекты комбинированной подачи нейтрального газа в конвертерную ванну. В кн.: Tр. IV Международного конгресса сталеплавильщиков. М.: Черметинформация, 1997. С. 104-107.

24. Протопопов Е.В., Чернятевич А.Г., Юдин С.В. Исследование химических и температурных градиентов в конвертерной ванне с использованием высокотемпературного моделирования // Известия вузов. Черная металлургия. 1997. № 10. С. 20-24.

25. Лушка А.И. Основы химической термодинамики и кинетики химических реакций. М.: Машиностроение, 1981. 240 с.

26. Продукты сгорания природного газа при высоких температуpax / И.Н. Карп, Б.С. Сорока, Л.Н. Дашевский, Д. Семернина. Киев: Техника, 1967. 381 с.

\section{СВЕДЕНИЯ ОБ АВТОРАХ}

Виталий Владимирович Солоненко, соискатель кафедры металлургии черных металлов, Сибирский государственный индустриальный университет

Евгений Валентинович Протопопов, д.т.н., профессор кафедры металлургии черных металлов, Сибирский государственный индустриальный университет

ORCID: 0000-0002-7554-2168

E-mail: protopopov@sibsiu.ru

Михаил Викторович Темлянцев, д.т.н., профессор кафедры теплоэнергетики и экологии, Сибирский государственный индустриальный университет

ORCID: 0000-0001-7985-5666

E-mail: uchebn_otdel@sibsiu.ru

Николай Филиппович Якушевич, д.т.н., профессор кафедры металлургии цветных металлов и химической технологии, Сибирский государственный индустриальный университет

E-mail: kafcmet@sibsiu.ru

Сергей Олегович Сафонов, ассистент кафедры металлургии черных металлов, Сибирский государственный индустриальный университет

E-mail: sergey.safonov.1950@mail.ru
21. Goodman N. Slag splashing of BOF converters. Iron and Steel Inst. 1996, no. XXX, pp. 24-33.

22. Diepmann D., Gharib M. The role of streamwise vortisity in the hear-field entrainment of round jets. Journal of Fluid Mechanics. 1992, vol. 245, pp. 643-668.

23. Protopopov E.V., Aizatulov R.S., Chernyatevich A.G. Technological aspects of combined supply of inert gas to converter bath. In: Papers of the IV International Congress of Steelmakers. Moscow: Chermetinformatsiya, 1997, pp. 104-107. (In Russ.).

24. Protopopov E.V., Chernyatevich A.G., Yudin S.V. Investigation of chemical and temperature gradients in a converter bath using hightemperature modeling. Izvestiya. Ferrous Metallurgy. 1997, no. 10, pp. 20-24. (In Russ.).

25. Lushka A.I. Fundamentals of Chemical Thermodynamics and Kinetics of Chemical Reactions. Moscow: Mashinostroenie, 1981, 240 p. (In Russ.).

26. Karp I.N., Soroka B.S., Dashevskii L.N., Semernina D. Products of Natural Gas Combustion at High Temperatures. Kiev: Tekhnika, 1967, 381 p. (In Russ.).

\section{INFORMATION ABOUT THE AUTHORS}

Vitalii V. Solonenko, Candidate for a degree of Cand. Sci. (Eng.) of the Chair of Ferrous Metallurgy, Siberian State Industrial University

Evgenii V. Protopopov, Dr. Sci. (Eng.), Prof. of the Chair of Ferrous Metallurgy, Siberian State Industrial University

ORCID: 0000-0002-7554-2168

E-mail: protopopov@sibsiu.ru

Mikhail V. Temlyantsev, Dr. Sci. (Eng.), Prof. of the Chair "Thermal Power and Ecology", Siberian State Industrial University ORCID: 0000-0001-7985-5666

E-mail: uchebn_otdel@sibsiu.ru

Nikolai F. Yakushevich, Dr. Sci. (Eng.), Prof. of the Chair "Non-Ferrous Metallurgy and Chemical Engineering", Siberian State Industrial University

E-mail: kafcmet@sibsiu.ru

Sergei O. Safonov, Assistant of the Chair of Ferrous Metallurgy, Siberian State Industrial University

E-mail: sergey.safonov.1950@mail.ru
Поступила в редакцию 26.11.2020

После доработки 22.12.2020

Принята к публикации 26.12.2020
Received 26.11.2020

Revised 22.12.2020

Accepted 26.12.2020 